\title{
Diskriminierung und Diskriminierungskritik: eine problemsoziologische Analyse
}

\author{
A. Scherr
}

Eingegangen: 29. März 2020 / Angenommen: 11. September 2020 / Online publiziert: 27. Oktober 2020

(C) Der/die Autor(en) 2020

Zusammenfassung Ein Verständnis von Diskriminierung als soziales Problem kann einen breiten gesellschaftlichen Konsens für sich beanspruchen. Denn es wird als ein Kennzeichen moderner, den normativen Prinzipien der Menschenrechte verpflichteter Gesellschaften angesehen, dass Diskriminierung aufgrund zugeschriebener oder tatsächlicher Kollektivmerkmale, z. B. aufgrund der Religionszugehörigkeit, des Geschlechts und der sexuellen Orientierung, aufgrund von Behinderungen oder von Ethnizität, als unzulässig gilt. Demgegenüber wird hier in einer problemsoziologischen Perspektive aufgezeigt, warum dieser Konsens fragwürdig und brüchig ist, sowie warum Diskriminierung auch nicht allein als Effekt tradierter Vorurteile und obsoleter Ideologien, sondern nur dann zureichend verstanden werden kann, wenn auch gesellschaftsstrukturelle Bedingungen von Diskriminierung und die Verwendung diskriminierender Unterscheidungen durch Organisationen berücksichtigt werden. Aufgezeigt werden damit Grundlagen und Perspektiven einer problemsoziologisch informierten Diskriminierungsforschung.

\footnotetext{
A. Scherr $(\bowtie)$

Institut für Soziologie, Pädagogische Hochschule Freiburg, Kunzenweg 21, 79117 Freiburg,

Deutschland

E-Mail: scherr@ph-freiburg.de
} 


\title{
Discrimination and Critique of Discrimination: an Analysis from the Perspective of the Sociology of Social Problems
}

\begin{abstract}
An awareness of discrimination as a social problem can claim a broad social consensus. For it is regarded as a characteristic of modern societies committed to the normative principles of human rights that discrimination on the basis of attributed or real collective characteristics, e.g. on the basis of religious affiliation, gender and sexual orientation, on the basis of disabilities or ethnicity, is considered unacceptable. In contrast to this, it is shown here why this consensus is questionable and fragile, as well as why discrimination cannot be understood solely as an effect of handed down prejudices and obsolete ideologies, but can only be understood sufficiently if socio-structural conditions of discrimination and the use of discriminatory distinctions by organisations are also taken into account. This will provide the basis and perspectives for research on discrimination that is informed by the sociology of social problems.

Wir glauben, dass ein besseres theoretisches Verständnis der sozialen Spannungen zu erwarten wäre, wenn Sozialwissenschaftler und Philosophen bereit wären, für eine Zeitlang ihre wohlgemeinte Vorstellung aufzugeben, dass Diskriminierung und andere soziale Übel ausschließlich in Vorurteilen gründen, die wie von Zauberhand verschwänden, sobald wir die Übeltäter davon in Kenntnis setzen, dass sie Vorurteile pflegen. (Schütz 2011 [1957], S. 215)
\end{abstract}

\section{Einleitung}

Sozialwissenschaftliche Diskriminierungsforschung setzt gewöhnlich voraus, dass es sich bei den unterschiedlichen Formen von Diskriminierung (etwa: Rassismus, Antisemitismus, Sexismus) zweifellos um soziale Probleme - um gesellschaftlich inakzeptable und veränderungsbedürftige Sachverhalte - handelt. Dementsprechend liegt der Fokus der einschlägigen Forschung auf der empirischen Erfassung des Ausmaßes von Diskriminierung und der Untersuchung der Ursachen und Gründe. Eine problemsoziologische Perspektive, die gesellschaftlich etablierte Problemdefinitionen selbst kritisch hinterfragt, ist dagegen bislang im Kontext der Diskriminierungsforschung kaum entwickelt worden; ihre Relevanz und ihr Potenzial sind auch nicht unmittelbar einleuchtend. Denn auch in der wissenschaftlichen Diskriminierungsforschung wird gewöhnlich vorausgesetzt, dass und weshalb Diskriminierung als soziales Problem zu betrachten ist. Im Unterschied dazu fordert die Problemsoziologie dazu auf, die Frage aufzuwerfen, warum es in modernen Gesellschaften als fraglos-selbstverständlich gilt, dass Diskriminierung generell ein soziales Problem ist, warum zugleich aber nur bestimmte Formen von Diskriminierung als problematisch gelten und andere nicht. Zu fragen ist weiter, wodurch und wie deren (Ent-)Problematisierung sozial bewerkstelligt wird sowie welche Deutungsrahmen damit etabliert werden. Damit, und dies wird im Weiteren noch näher zu erläutern sein, kann die Problemsoziologie zur Entwicklung einer reflexiven Diskriminierungsforschung beitragen, die Distanz gegenüber gesellschaftlich institutionalisierten Problemdefini- 
tionen einnimmt und dadurch zu einem kritischen Blick auf deren Voraussetzungen, Blindstellen und Implikationen in der Lage ist.

Zwar ist der Diskriminierungsforschung diesbezüglich keine gänzliche Naivität vorzuwerfen. So wurden und werden im Kontext der Diskriminierungsforschung bestimmte Problemdefinitionen wissenschaftlich und politisch kontrovers diskutiert, so z.B., ob Formen der Feindseligkeit gegen Migranten und Minderheiten angemessener als Ausländer- bzw. Fremdenfeindlichkeit oder aber als Rassismus zu fassen sind (siehe dazu etwa Friese 2019); bereits Anfang der 1990er-Jahre wurde zugleich eine inflationäre, allzu unspezifische Verwendung des Rassismusbegriffs kritisiert (Heitmeyer 1992; vgl. Scherr 2017b). Zudem ist mit der Unterscheidung von struktureller, institutioneller und interaktioneller Diskriminierung (Hormel und Scherr 2004, 2010; Gomolla und Radtke 2012; Rommelspacher 1995; Scherr 2017a) darauf hingewiesen worden, dass die gängige Gleichsetzung von Diskriminierung mit stigmatisierenden Sprechakten und absichtsvoll benachteiligenden Handlungen nicht tragfähig ist. Eine darüber hinausgehende problemsoziologische Auseinandersetzung mit dem Diskriminierungsbegriff - und nicht nur mit den Semantiken und Vorannahmen, die zur Problematisierung bestimmter Formen von Diskriminierung wissenschaftlich und/oder politisch verwendet werden - ist dagegen bislang nicht erfolgt.

Darauf, dass die gesellschaftliche Problematisierung von Diskriminierung selbst einer kritischen Betrachtung zu unterziehen ist, hatte jedoch bereits Parkin in einem zuerst 1974 publizierten Aufsatz hingewiesen (Parkin 1984). Er akzentuiert, dass die gesellschaftliche Problematisierung von Diskriminierung nicht als eine generelle Infragestellung sozialer Ungleichheiten verstanden werden kann, sondern nur als Infragestellung eines sozialen Mechanismus, durch den Positionszuweisungen in der Struktur sozialer Ungleichheiten bewerkstelligt und gerechtfertigt werden. Sein Zweifel daran, ob ein Übergang von zugeschriebenen Gruppenmerkmalen zu individuellen Leistungsmerkmalen als Unterscheidungskriterium - also von Diskriminierung zu meritokratischer Auslese - tatsächlich als ein moralischer Fortschritt zu bewerten sei, hat jedoch keine Resonanz gefunden (Parkin 1984, S. 126). Die Selbstverständlichkeit, mit der angenommen wird, dass ohne weitere theoretische Klärungen davon ausgegangen werden kann, dass Diskriminierung ein soziales Problem ist, wird auch darin sichtbar, dass in einschlägigen Beiträgen zum „Handbuch soziale Probleme" (Albrecht und Groenemeyer 2012) vorausgesetzt wird, dass Diskriminierung (als ethnische Diskriminierung und Frauendiskriminierung) auf ein soziales Problem verweist, dort aber keine Klärung dazu erfolgt, warum Diskriminierung in diesen Fällen als ein soziales Problem zu betrachten ist und was das soziale Problem Diskriminierung von anderen sozialen Problemen (wie z. B. abweichendes Verhalten, Kriminalität oder Sucht) unterscheidet.

Auf der Grundlage einer anhaltenden Auseinandersetzung mit den Grundlagen einer eigenständigen soziologischen Diskriminierungsforschung (Scherr 2014a, 2017a) werden im Folgenden Überlegungen entwickelt, die dazu beitragen sollen, dieses Reflexionsdefizit zu verringern und Perspektiven einer problemsoziologischen Diskriminierungsforschung aufzuzeigen. 


\section{Gesellschaftsstrukturelle Bedingungen und menschenrechtliche Begründungen der Anerkennung von Diskriminierung als soziales Problem}

Für das Selbstverständnis moderner Gesellschaften als Gesellschaften freier und gleicher Individuen ist die Ablehnung von Diskriminierung von konstitutiver Bedeutung. Denn erst dadurch, dass auf die Ungleichbehandlung von Personen aufgrund zugeschriebener oder tatsächlicher Kollektivmerkmale verzichtet, oder dies zumindest als ein gesellschaftlich akzeptiertes Prinzip deklariert wird, werden Gesellschaftsmitglieder zu Individuen, die gleiche Rechte und eine Anerkennung ihrer Würde beanspruchen können. Dementsprechend ist in der Philosophie der Menschenrechte überzeugend argumentiert worden, dass das im Artikel 2 der Allgemeinen Erklärung der Menschenrechte verankerte Diskriminierungsverbot einen systematisch anderen Stellenwert hat als eine Reihe anderer menschenrechtlicher Normen: Demnach ist das Diskriminierungsverbot ,nicht nur eine Norm neben anderen, sondern hat darüber hinaus den Stellenwert eines Strukturprinzips“, da Rechte nur dadurch zu Menschenrechten werden, die für alle gelten, dass sie ,nach dem Grundsatz der Nicht-Diskriminierung gewährleistet werden“ (Bielefeldt 2010, S. 23). Würden diese Rechte dagegen an bestimmte Voraussetzungen, z.B. an bestimmte individuelle Merkmale oder die Zugehörigkeit zu einer bestimmten sozialen Gruppe gebunden, dann „wären diese Rechte keine Menschenrechte, sondern lediglich Privilegien“ (ebd.; vgl. Margalit 1998).

In der Soziologie der Menschenrechte ist in Übereinstimmung damit akzentuiert worden, dass der Menschenrechtsdiskurs eine Sozialontologie voraussetzt, in der Individuen (und nicht Kollektive wie Familien, Verwandtschaften, Stämme, Ethnien usw.) als Grundeinheiten des Sozialen und als zentrale soziale Akteure gelten (Koenig 2015, S. 105 ff.). Die gesellschaftsstrukturelle Grundlage, die dem Selbstverständnis moderner Gesellschaften als einer „Gesellschaft der Individuen“ (Elias 1991) Plausibilität verleiht, ist in der soziologischen Klassik bei Karl Marx als Vergesellschaftung von Individuen (und nicht von Familienhaushalten) als Träger der Ware Arbeitskraft beschrieben worden und bei Emile Durkheim als Individualisierung durch fortschreitende gesellschaftliche Arbeitsteilung (vgl. Beck 1984). Darauf Bezug nehmend hat Luhmann (1993a) argumentiert, dass für die Struktur funktionaler Differenzierung eine Form der Vergesellschaftung durch Inklusions- bzw. Exklusionsprozesse charakteristisch ist, durch die Individuen (und nicht Familien oder Verwandtschaften) in je spezifischer und selektiver Weise in gesellschaftliche Funktionssysteme und Organisationen einbezogen bzw. von diesen ausgeschlossen werden. Kennzeichnend für moderne Gesellschaften ist demnach die Zurückdrängung der Bedeutung partikularer sozialer Bindungen und Verpflichtungen und damit eine Vergesellschaftung von Personen als Individuen - als individuelle Arbeitskräfte, Rechtssubjekte, Konsument*innen, Wähler*innen usw. Dem korrespondiert die soziologische Annahme einer „Kontingenz sozialer Zugehörigkeiten“ (Hirschauer 2014, S. 170).

Die zentralen kulturellen Entsprechungen dieses strukturellen Individualismus bestehen in bürgerlich-demokratischen Gesellschaften erstens in der Vorstellung, dass die Zuweisung gesellschaftlicher Positionen das Ergebnis eines meritokratischen 
Wettbewerbs unter Bedingungen der Chancengleichheit sein soll sowie zweitens in der Forderung, dass alle Individuen beanspruchen können, über gleiche Rechte zu verfügen und Anspruch auf Anerkennung einer Würde zu haben, die ihnen - im Unterschied zu ständischen Ehre - als Individuen zukommt. Darauf bezogen haben Heintz (2015) und Japp (2015) argumentiert, dass von einem wechselseitigen Bedingungszusammenhang von Menschenrechten und funktionaler Differenzierung auszugehen sei: Funktionale Differenzierung setzt demnach einerseits voraus, dass Bindungen an partikulare Gruppen zurückgedrängt und Individuen dadurch befähigt werden, sich an den Anforderungen und Verhaltenswartungen der Funktionssysteme und Organisationen auszurichten. So betrachtet stellt „die soziale Institutionalisierung der Grundrechte“ als Individualrechte - und das für diese konstitutive Diskriminierungsverbot - einerseits eine „Folgeerscheinung funktionaler Differenzierung“ dar (Heintz 2015, S. 47). Andererseits aber kann plausibel angenommen werden, dass die Durchsetzung der Menschenrechte eine historische und systematische Voraussetzung funktionaler Differenzierung ist, da erst dadurch die Koppelung von Karrieren an individuelle Bildungsbiografien, die ökonomische Vergesellschaftung individueller Arbeitskräfte, die Adressierung von Individuen als Rechtssubjekte und die Freisetzung individueller Lebensstile aus religiösen oder politischen Vorgaben möglich wird. Insofern kann mit einiger Plausibilität eine grundlegende Wechselwirkung angenommen werden:

Wer funktionale Differenzierung haben will, muss gleichzeitig für die Umsetzung der Menschenrechte sorgen, und wer sich für deren Umsetzung einsetzt, transportiert damit, willentlich oder nicht, auch eine spezifische Gesellschaftsform. (Heintz 2015, S. 48)

Diese abstrakt gefassten Überlegungen legen es nahe, funktionale Differenzierung sowie die politische und rechtliche Gewährleistung von Menschenrechten als sich wechselseitig bedingende Prozesse in den Blick zu nehmen, die sich in jeweiligen nationalgesellschaftlichen Kontexten mehr oder weniger umfassend und konsequent durchsetzen. Denn es ist keineswegs plausibel davon auszugehen, dass die funktionale Ausdifferenzierung einer kapitalistischen Marktökonomie notwendig mit der Durchsetzung von Rechtsstaatlichkeit, Religionsfreiheit und demokratischen Rechten einhergeht, dass sich funktionale Differenzierung also zwingend in einer Weise realisiert, die alle gesellschaftlichen Teilbereiche umfasst sowie durch die umfassende Gewährleistung von Menschenrechten abgestützt ist. Vor diesem Hintergrund ist festzustellen, dass in den Gleichbehandlungsrichtlinien der Europäischen Union (Antirassismusrichtlinie 2000/43/EG; Rahmenrichtlinie Beschäftigung 2000/78/EG; Richtlinie zur Verwirklichung des Grundsatzes der Gleichbehandlung von Männern und Frauen 2002/73 EG) sowie im deutschen Allgemeinen Gleichbehandlungsgesetz von 2006 eine politische und rechtliche Anerkennung von Diskriminierung als gesellschaftliches Problem zum Ausdruck kommt. Für diese Entwicklung gilt, dass sie zwar gesellschaftsstrukturell ermöglicht, aber keine selbstläufige Konsequenz des strukturellen Individualismus ist. Vielmehr handelt es sich um eine politisch und rechtlich sehr voraussetzungsvolle Folge einer Entwicklung, die durch die weltgesellschaftliche Bedeutungszunahme und Institutionalisierung des Menschenrechtsdiskurses seit den 1970er-Jahren (Eckel 2009; Eckel und Moyn 2012) sowie durch 
die Inanspruchnahme der Menschenrechte als zentraler Bezugspunkt eines Selbstverständnisses der Europäischen Union als Wertegemeinschaft gekennzeichnet ist.

Sozialhistorische Studien zeigen diesbezüglich auf, dass seit den 1970er-Jahren eine durch unterschiedliche Entwicklungen ausgelöste Bedeutungszunahme des Menschenrechtsdiskurses erfolgte, eine „Menschenrechtsrevolution“ (Ignatieff 2002, S. 30), die dazu geführt hat, dass nationale Politik veranlasst ist, sich menschenrechtlich zu legitimieren und internationale politische Kontroversen zunehmend in der Sprache der Menschenrechte ausgetragen werden (Eckel 2009, S. $458 \mathrm{ff}$.). Da ein explizites und weit gefasstes Diskriminierungsverbot im Artikel 3 der Allgemeinen Erklärung der Menschenrechte von 1948 verankert ist, führt dies auch dazu, dass das Diskriminierungsverbot im Kontext der politischen Aufwertung des Menschenrechtsdiskurses als quasi selbstverständliche Grundlage für Problematisierung und Kritik beansprucht werden kann. Zudem ist dieses ohnehin - wie gezeigt (s. oben) - für ein modernes Verständnis von Menschenrechten als Rechten, die Individuen zukommen, konstitutiv.

In einer fortschrittsoptimistischen Perspektive ließe sich die skizzierte Entwicklung als ein Prozess interpretieren, der schrittweise zu einer politisch und rechtlich einflussreichen Kritik tradierter Formen von Diskriminierung geführt hat: Diskriminierende Kategorien (prominent im Fall von „Rasse“) bzw. ihre benachteiligende Verwendung (prominent im Fall von Geschlecht) wurden - vorangetrieben durch soziale Bewegungen, wissenschaftliche und politische Diskurse - kritisiert und haben in der Folge mehr oder weniger weitreichend an Bedeutung verloren. Diskriminierungskritik stellt sich in dieser Perspektive als Bestandteil eines unabgeschlossenen Prozesses dar, dessen angestrebtes und langfristig zu erwartendes Ergebnis eine umfassende normative Delegitimierung und praktische Überwindung aller Formen von Diskriminierung sein soll.

\section{Keine geradlinige Fortschrittsgeschichte}

Zweifellos sind diesbezüglich zwar erhebliche Fortschritte im Sinne einer gesellschaftlichen Anerkennung von Diskriminierung als soziales Problem festzustellen. Dies gilt u. a. für die rechtliche Verankerung von Diskriminierungsverboten im europäischen und nationalen Recht, den Verzicht auf Pathologisierung und rechtliche Sanktionierung von Homosexualität, sowie einen Bewusstseinswandel, der dazu geführt hat, dass die Existenz von Rassismus als auch gegenwärtig noch relevante Form von Diskriminierung im politischen Diskurs anerkannt wird. ${ }^{1}$ Auf der Grundlage der Theorien und der empirischen Befunde sozialwissenschaftlicher Diskriminierungsforschung sind jedoch substanzielle Einwände gegen eine solche fortschrittsoptimistische Sichtweise geltend zu machen: Sozialhistorische Studien haben nachgewiesen, dass Inanspruchnahmen von Menschenrechten und normativen Diskriminierungsverboten immer auch von machtpolitischen Interessenlagen überformt sowie Gegenstand sozialer Konflikte zwischen Mehrheiten und Minderheiten waren (Davidson

\footnotetext{
1 Für Informationen zu den Entwicklungen in diesen und anderen Bereichen siehe die einschlägigen Beiträge in Scherr et al. (2017).
} 
2012; Eckel 2009; Eckel und Moyn 2012). In Studien der Rassismusforschung wurde mit dem Begriff der ,,past-in-present-discrimination“ (Feagin und Eckberg 1980) darauf hingewiesen, dass gegenwärtige Formen rassistischer Diskriminierung nur dann angemessen verstanden werden können, wenn die Auswirkungen vergangener Diskriminierung auf die gegenwärtige soziale Situation der davon Betroffenen berücksichtigt wird; Auswirkungen, die auch dann fortbestehen, wenn die ideologischen Grundlagen vergangener Diskriminierung überwunden sind und direkte absichtsvolle Diskriminierung gesellschaftlich sanktioniert wird. Soziologische Diskriminierungstheorien haben zudem aufgezeigt, dass Diskriminierung keineswegs zureichend als Effekt der Tradierung unzeitgemäßer Ideologien und Vorurteile begriffen werden kann: Soziologisch betrachtet resultiert Diskriminierung auch aus der diskursiven Tradierung und gesellschaftsstrukturellen, insbesondere politischen und rechtlichen, Verankerung kategorialer Unterscheidungen sowie ihrer Verwendung durch Organisationen bei der Herstellung, Begründung und Rechtfertigung von Zugangsregulierungen, Positionszuweisungen und der Leistungserbringung (Gomolla 2017; Scherr 2017a; Tilly 1998). Diskriminierung durch Organisationen, z. B. durch Betriebe bei Personalentscheidungen, ist dabei nicht allein die Folge von Vorurteilen und benachteiligenden Absichten, sondern u. a. ein Resultat betriebswirtschaftlicher Kalküle sowie der Notwendigkeit, Akzeptanz für jeweilige Entscheidungen sowie für Maßnahmen der (Un-)Gleichbehandlung in der sozialen Umwelt von Betrieben rechtfertigen zu müssen. Diskriminierung in Schulen bei der Ermöglichung von Bildungskarrieren ist nicht bzw. nicht zureichend als Ergebnis von Stereotypen oder benachteiligenden Absichten erklärbar. Eine zentrale Bedeutung kommt dabei auch der Einschätzung von Lehrkräften bezüglich der Unterstützungsmöglichkeiten zu, über die Schulen und Familien, z. B. bei Schüler*innen nicht-deutscher Muttersprache oder mit psychischen Beeinträchtigungen, verfügen. ${ }^{2}$ Diskriminierung kann dann auch das Ergebnis der wohlmeinenden Absicht sein, jeweilige Schüler*innen und ihre Familien nicht zu überfordern.

Diskriminierende Unterscheidungen sind für die Herstellung, Aufrechterhaltung und Rechtfertigung von sozioökonomischen Ungleichheiten, Machtasymmetrien und Statushierarchien funktional, da sie Eigenschaftszuschreibungen umfassen, die den Angehörigen einer diskriminierten sozialen Gruppe die Fähigkeit und das Interesse bestreiten, privilegierte Position einzunehmen, oder mit der normativen Überzeugung einhergehen, dass diese die Position des gleichberechtigten Gesellschaftsmitglieds nicht legitim beanspruchen können. Soziologisch betrachtet ist die Verwendung diskriminierender Unterscheidung deshalb unter Bedingungen sozialer Ungleichheiten eine Möglichkeit, Optionen sozialer Teilhabe und Positionszuweisungen zu regulieren und zu rechtfertigen. Tilly (1998, S. 7) spitzt diese Perspektive zu der These zu, dass dauerhafte und gravierende soziale Ungleichheiten eine notwendige Entsprechung in ,categorial differences such als black/white, male/female, citizen/foreigner, or Muslim/Jew“ benötigen, da diese für die Aufrechterhaltung von

\footnotetext{
2 Ausführlicher ist dies für schulische Diskriminierung bei Gomolla und Radtke (2012) sowie Hormel (2007) dargestellt, für betriebliche Diskriminierung u. a. bei Imdorf und Scherr (2015), Imdorf (2017) und Scherr et al. (2015).
} 
Privilegierungen durch soziale Schließung bzw. Exklusion sowie Ausbeutung unverzichtbar sind.

Mit diesen Hinweisen war zu verdeutlichen, dass Diskriminierung nicht zureichend als ein Effekt unzeitgemäßer Ideologien und irrationaler Vorurteile verstanden werden kann, deren Attraktivität sozialpsychologisch - als Folge von Gruppenkonflikten (Tajfel 1982) bzw. einer Gemengelage von Abstiegsängsten, Ausgrenzungserfahrungen und autoritären Dispositionen - erklärt werden kann (u. a. Heitmeyer 2012; Legge und Mansel 2012, S. 497 ff.; Möller und Neuscheler 2018). Die menschenrechtlich verankerte, politische und rechtliche Anerkennung von Diskriminierung als soziales Problem kann deshalb nicht sinnvoll zu einer Beschränkung der sozialwissenschaftlichen Diskriminierungsforschung auf die empirische Vermessung und Erklärung von Vorurteilen und Formen der sog. gruppenbezogenen Menschenfeindlichkeit führen. Historische und soziologische Diskriminierungsforschung fordern demgegenüber dazu auf, die gesellschaftlichen Prozesse und Strukturen zu untersuchen, die zu einer Verschränkung von Machtasymmetrien, sozioökonomischen Ungleichheiten und Prestigehierarchien mit Gruppenkategorien geführt haben (Hálfdarnason und Vilhelmsson 2017; Scherr 2017a). Erst vor diesem Hintergrund kann auch verstanden werden, welche Stereotype und Vorurteile in einer Gesellschaft verbreitet sind. Der spezifische Beitrag einer problemsoziologischen Perspektive, liegt dabei darin, die Prozesse zu analysieren, durch die bestimmte Formen von Stigmatisierung und Benachteiligung mehr oder weniger erfolgreich zu Ausprägungen unzulässiger Diskriminierung erklärt werden - und andere nicht.

\section{Diskriminierung als soziales Problem}

In Bezug auf ein soziales Phänomen von Diskriminierung zu sprechen, impliziert in modernen Gesellschaften, die beanspruchen, sich auf die Menschenrechte als Wertegrundlage zu beziehen, per se den Anspruch, damit ein soziales Problem (im Sinne gesellschaftlich nicht akzeptabler Strukturen oder Praktiken) zu benennen, das einen ,anerkannten, offiziellen Status“ (Gronemeyer 2012, S. 89) hat oder für das ein solcher eingefordert wird. Dem entspricht, dass die sozialwissenschaftliche Diskriminierungsforschung ihren Fokus darin hat, a) Ausmaß, Ausprägungen und Auswirkungen von Diskriminierung in gesellschaftlichen Teilsystemen (z. B. schulische Diskriminierung, Diskriminierung auf Arbeits- und Wohnungsmärkten oder durch die Polizei) oder b) im Fall davon betroffener Minderheiten (z. B. Afrodeutsche, Personen mit zugeschriebenem Migrationshintergrund, Homosexuelle, Muslime, Sinti und Roma) empirisch zu erfassen, c) Analysen der Ursachen und Gründe vorzunehmen sowie auf dieser Grundlage d) Vorschläge zur praktischen Überwindung zu unterbreiten (ADS 2017; Bertelsmann Stiftung 2018; Scherr et al. 2017). Eine solche Forschung ist anschlussfähig an die Informationsbedarfe institutioneller Akteure der Antidiskriminierungspolitik und wird zu einem relevanten Teil auch als politische Auftragsforschung betrieben. ${ }^{3}$ Diskriminierungsforschung ist insofern überwiegend solche Forschung, die für politisches und rechtliches Vorgehen gegen

\footnotetext{
3 Bedeutsamster institutioneller Akteur auf nationaler Ebene ist die Antidiskriminierungsstelle des Bundes (www.antidiskriminierungsstelle.de), auf europäischer Ebene die European Agency for Fundamental
} 
Diskriminierung sowie mediale Thematisierungen relevante Einsichten zur Verfügung stellen sowie zur Unterstützung zivilgesellschaftlicher Akteure und zur Qualifizierung professionellen Handelns beitragen soll. Dass Diskriminierung ein soziales Problem ist bzw. dass jeweils in Rede stehende Phänomene (wie z. B. Rassismus) sinnvoll als Diskriminierung zu problematisieren sind, wird dabei in der Regel als fraglose Prämisse vorausgesetzt. Dies unterscheidet eine theoretisch unreflektierte, auf die Produktion politisch und medial relevanter Daten ausgerichtete empirische Diskriminierungsforschung ersichtlich von einer solchen problemsoziologisch informierten soziologischen Forschung, die gesellschaftlich etablierte Problemkategorien (wie z. B. Kriminalität, Sucht oder Verwahrlosung) nicht voraussetzt, sondern diese selbst als Gegenstand wissenschaftlicher Analyse begreift und kritisch in den Blick nimmt.

Eine darauf ausgerichtete problemsoziologische Wendung der Diskriminierungsforschung scheint zunächst wenig plausibel zu sein. Aber auch in diesem Fall gilt, dass der erste Eindruck täuschen kann. Denn auch im Fall des sozialen Problems Diskriminierung kann gezeigt werden, dass im Anti-Diskriminierungsdiskurs eine nicht-beliebige, voraussetzungsvolle, selektive und dadurch folgenreiche Perspektive der (Ent-)Problematisierung eingenommen wird.

\subsection{Problematisierungen als Ergebnis gesellschaftlich bedingter Konstruktionsprozesse}

Die Forderung nach einer problemsoziologisch aufgeklärten reflexiven Diskriminierungsforschung provoziert den Einwand, dass davon kein für politische, rechtliche und pädagogische Maßnahmen gegen Diskriminierung nützliches Wissen über Ursachen und Ausprägungen zu erwarten ist, sondern nur wenig hilfreiche Überlegungen dazu, dass das soziale Problem Diskriminierung, wie andere soziale Probleme auch, Ergebnis von Konstruktionsprozessen ist. Dieser Einwand ist jedoch nur dann plausibel, wenn eine grundsätzliche Unvereinbarkeit zweier alternativloser Paradigmen unterstellt wird: einer theoretisch naiven Ätiologie einerseits und eines radikalen Konstruktivismus andererseits, der auf Annahmen über die reale Problematik der jeweils problematisierten Sachverhalte verzichtet. Trotz aller Kontroversen dazu, was die paradigmatischen Grundlagen einer eigenständigen Soziologie sozialer Probleme sind oder sein sollten (Groenemeyer 2012, S. $17 \mathrm{ff}$.), kann demgegenüber m. E. ein produktiver Kern der Problemsoziologie bestimmt werden, der diese vermeintlich alternativlose Entgegensetzung zweier konkurrierender Paradigmen vermeidet (vgl. Groenemeyer 2012, S. 17 ff.; Scherr 2002, 2019). Denn es ist keineswegs erforderlich, den realen Problemcharakter eines sozialen Sachverhalts, der gesellschaftlich als soziales Problem gilt, zu bestreiten, um die Perspektive eines kritischen Sozialkonstruktivismus einnehmen zu können, der nach den hoch voraussetzungsvollen und selektiven Prozessen fragt, durch die bestimmte Sichtweisen sozialer Probleme etabliert und durchgesetzt werden. Im Unterschied zu einem in der angewandten Sozialforschung zunehmenden einflussreichen theorielosen Empirismus, der noch

Rights (https://fra.europa.eu/); hinzu kommen einflussreiche Stiftungen sowie vielfältige Institutionen und Initiativen auf regionaler und lokaler Ebene. 
hinter den Diskussionsstand des alt-ehrwürdigen Positivismusstreits und des kritischen Rationalismus zurückfällt, setzt sozialwissenschaftliche Problemsoziologie die Problemwahrnehmungen gesellschaftlicher (politischer, medialer, rechtlicher, pädagogischer usw.) Institutionen und Akteure jedoch nicht schlicht als zutreffende Problemdefinitionen voraus. Vielmehr wird versucht, dadurch Erkenntnisgewinne zu erzielen, dass deren Entstehung, Durchsetzung und Funktion sowie deren Folgen analysiert werden. Es geht folglich um die distanzierte und schon dadurch kritische Betrachtung jeweiliger selektiver Problemkonstruktionen sowie der gesellschaftlichen Bedingungen, unter denen diese als mehr oder weniger plausible Sichtweisen dargestellt werden können.

Nicht nur, aber auch im Fall von Diskriminierung sind diesbezüglich gesellschaftliche Machtverhältnisse zu berücksichtigen, die auch Machtasymmetrien in Hinblick auf die Definitions- und Deutungsmacht sozialer Probleme sind (dazu zusammenfassend Groenemeyer 2012, S. 73 ff.). Der analytische Rekurs auf Machtverhältnisse, darin verankerte Interessen und Konflikte - wie ihn etwa Steinert (1981) in seiner Kritik der funktionalistischen Problemsoziologie vehement eingefordert hat - ist folglich zwar unverzichtbar. Er ist aber unzureichend, um die Chancen der Etablierung und Durchsetzung jeweiliger Problematisierungen erklären zu können. Denn Machtasymmetrien sind selbst Ausdruck gesellschaftlicher Strukturen: Unter Bedingungen einer komplex differenzierten Gesellschaft bedeutet dies nicht zuletzt, dass die Annahme einer singulären Machtquelle und dem entsprechend einer singulären definitionsmächtigen Akteursgruppe (vulgo: herrschenden Klasse) obsolet ist. Vielmehr muss erstens mit einer Pluralität von teilsystemischen Perspektiven gerechnet werden, die zu konkurrierenden (nicht zuletzt: politischen, rechtlichen, religiösen, pädagogischen, wissenschaftlichen) Relevanzen der Problematisierung unterschiedlicher Phänomene sowie zu unterschiedlichen Problematisierungsweisen eines Phänomens führen. Zudem ist davon auszugehen, dass unter Bedingungen kultureller Pluralisierung heterogene normative Deutungshorizonte, nicht zuletzt konkurrierende politische Ideologien und religiöse Glaubensgewissheiten, für jeweilige (Ent-)Problematisierungen beansprucht werden. Es ist auch zu berücksichtigen worauf insbesondere im Kontext des Neo-Institutionalismus hingewiesen worden ist (Meyer et al. 2005, S. 28 ff.; Koenig 2015, S. 99 ff.) - dass jede gesellschaftliche Problemkommunikation einen geteilten (impliziten oder expliziten) kognitiven und moralischen Referenzrahmen voraussetzt, damit Kommunikation über mehr oder weniger zutreffende Realitätsbeschreibungen und potenziell kontroverse Bewertungen möglich wird. In der Perspektive einer sozialhistorisch informierten Soziologie sind zudem dynamische Wechselwirkungen zwischen Gesellschaftsstruktur und Semantik zu berücksichtigen (Luhmann 1993b, S. 9 ff.).

Damit sind einige Fragestellungen für eine problemsoziologisch aufgeklärte Diskriminierungsforschung skizziert. Diese können im Weiteren nicht umfassend bearbeitet werden. Fokussiert werden hier insbesondere die strukturellen Ermöglichungsbedingungen bestimmter Formen der Problematisierung sowie die damit einhergehenden Ausblendungen. 


\subsection{Abstraktion und Respezifizierung}

Die skizzierte problemsoziologische Perspektive fordert dazu auf, Diskriminierung als einen spezifischen Modus der Problematisierung in den Blick zu nehmen. Für diesen ist zunächst kennzeichnend, dass die erfolgreiche Bezeichnung eines Sachverhalts als Form von Diskriminierung innerhalb des Referenzrahmes, der durch den hegemonialen Bezug auf die Menschenrechte gegeben ist, nicht hinterfragt werden kann: Innerhalb dieses Referenzrahmens kann nicht bestritten werden, dass Diskriminierung prinzipiell abzulehnen ist, sondern nur, ob es sich beim jeweiligen Sachverhalt tatsächlich um Diskriminierung handelt, oder aber ob Ausnahmetatbestände vorliegen, die zwar die Möglichkeit einer normativen Kritik nicht aushebeln, aber die Möglichkeit einer rechtlichen Sanktionierung einschränken. ${ }^{4}$ Wer dagegen die Überzeugung in Anspruch nimmt, dass Diskriminierung (oder bestimmte Formen davon wie rassistische oder geschlechtsbezogene) wünschenswert ist und zulässig sein soll, stellt sich außerhalb des menschenrechtlichen Referenzrahmens und wird entsprechend als Außenseiter (z.B. Rassist, Rechtsextremer, Sexist) markiert, mit dem eine sinnvolle Kommunikation kaum mehr möglich ist.

Ein weiteres Merkmal der menschenrechtlich gerahmten Problematisierung von Diskriminierung besteht darin, dass Diskriminierung eine abstrahierende Oberkategorie ist, die in ihrer historischen Genese, gesellschaftlichen Verankerung und in ihren Auswirkungen durchaus unterschiedliche Phänomene umfasst. Diese Abstraktion erfordert politisch, rechtlich, pädagogisch und auch wissenschaftlich dann Respezifizierungen, wenn bei der Analyse jeweiliger Formen von Diskriminierung und darauf bezogenen Praktiken nicht von den Unterschieden jeweiliger Formen von Diskriminierung abgesehen werden kann bzw. soll - von Unterschieden zum Beispiel zwischen biologischem Rassismus und religiösem Antisemitismus, oder etwa zwischen geschlechtsbezogener und altersbezogener Diskriminierung. Dieses Erfordernis der Respezifizierung ermöglicht zugleich eine Hierarchisierung der Bedeutung, die jeweiligen Diskriminierungsmerkmalen zugesprochen wird, ihrer Dringlichkeit als soziales Problem, und etabliert auch die Möglichkeit einer Selektivität bei der politischen und rechtlichen Berücksichtigung bestimmter Diskriminierungstatbestände.

Die Relevanz dieser Überlegungen kann zunächst anhand zentraler Dokumente des menschenrechtlichen und juristischen Anti-Diskriminierungsdiskurses verdeutlicht werden: In der Allgemeinen Erklärung der Menschenrechte ist eine generelle Ablehnung von Diskriminierung in Verbindung mit einer offen gehaltenen Liste möglicher Diskriminierungsmerkmale formuliert: Demnach hat jede*r „Anspruch auf alle in dieser Erklärung verkündeten Rechte und Freiheiten“ und dies ,ohne irgendeinen Unterschied, etwa nach Rasse, Hautfarbe, Geschlecht, Sprache, Religion, politischer oder sonstiger Anschauung, nationaler oder sozialer Herkunft, Vermögen,

\footnotetext{
${ }^{4}$ Diesbezügliche Analysen des Diskriminierungsrechts zeigen u. a. auf, dass dort für unterschiedliche Diskriminierungstatbestände relevante Rechtfertigungsgründe vorgesehen sind, dass der Geltungsbereich jeweiliger Regelungen beschränkt ist - so erstreckt sich z. B. der Geltungsbereich des Allgemeinen Gleichbehandlungsgesetzes zwar auch auf den öffentlichen Dienst als Arbeitgeber, nicht aber auf die Leistungserbringung im Verhältnis von Staat und Bürger*innen - sowie dass die Möglichkeiten der wirksamen Durchsetzung des Antidiskriminierungsrechts begrenzt sind; siehe dazu etwa Pärli (2017).
} 
Geburt oder sonstigem Stand“ (AEDM, Art. 2, Hervorhebung A. S.). Damit wird im Sinne eines normativen Grundsatzes - Juristen sprechen diesbezüglich von soft law - ein uneingeschränktes Diskriminierungsverbot formuliert, das die Möglichkeit der Hinzufügung weiterer Diskriminierungskategorien vorsieht. ${ }^{5}$

In den als unmittelbares Recht, auf das sich Betroffene im Klageverfahren vor Gerichten berufen können, wirksamen Bestimmungen des europäischen und deutschen Diskriminierungsrechts erfolgt demgegenüber zwar einerseits eine sozialwissenschaftlich informierte Präzisierung des Diskriminierungsbegriffs: Dort wird zwischen unmittelbarer Diskriminierung, d.h. einer Ungleichbehandlung durch Personen, sowie mittelbarer Diskriminierung unterschieden, die dann vorliegt, ,wenn dem Anschein nach neutrale Vorschriften, Kriterien oder Verfahrenen wegen eines in $\S 1$ genannten Grundes gegenüber anderen Personen in besonderer Weise benachteiligen können“, so die dem europäischen Recht entsprechende Formulierung des Allgemeinen Gleichbehandlungsgesetz (AGG, §3). Damit wird hier der Einsicht der Forschung über institutionellen Rassismus und organisationale Diskriminierung (siehe zusammenfassend Gomolla 2017) Rechnung getragen, dass Diskriminierung durch Organisationen gerade nicht zureichend durch benachteiligende Absichten und Handlungen erklärt werden kann. ${ }^{6}$ Andererseits aber impliziert der hier vorgenommene Verweis auf $\S 1$ eine weitreichende Einschränkung. Denn als rechtlich sanktionierbare Diskriminierung gelten dort allein „Benachteiligungen aus Gründen der Rasse oder wegen der ethnischen Herkunft, des Geschlechts, der Religion oder Weltanschauung, einer Behinderung, des Alters oder der sexuellen Identität"“. Dass die Offenheit des Diskriminierungsverständnisses der Allgemeinen Menschenrechte hier zurückgenommen wird und dort genannte Diskriminierungskategorien wie nationale oder soziale Herkunft nicht (mehr) als relevante Diskriminierungskategorien genannt werden, verweist auf eine erklärungsbedürftige Selektivität. Die generelle, von jeweiligen Ausprägungen abstrahierende Ablehnung von Diskriminierung transformiert sich damit in eine selektive politische und rechtliche Spezifizierung von Verboten bestimmter Formen von Diskriminierung, in denen die Abstraktion zurückgenommen wird, die dem menschenrechtlichen Verbot jeder Diskriminierung zugrunde liegt.

\subsection{Diskriminierung und soziale Ungleichheiten}

Diese Selektivität betrifft nicht zuletzt die absichtsvolle Ausklammerung von Staatsangehörigkeit aus der Liste der Diskriminierungsmerkmale. Diesbezüglich ist festzustellen, dass das Recht von Staaten, in benachteiligender Weise zwischen Staatsangehörigen und Ausländer*innen zu unterscheiden, als konstitutives Prinzip staatlicher Souveränität gilt. Dies betrifft Ansprüche auf wohlfahrtsstaatliche Leistungen, die Staatsangehörigen vorbehalten sind, Zugangsregulierungen zum Arbeitsmarkt, die Staatsangehörige privilegieren, und nicht zuletzt auch das Recht von Staaten, über

\footnotetext{
5 Allerdings gilt dieses Diskriminierungsverbot nur für die Rechte und Freiheiten, die in der Allgemeinen Erklärung der Menschenrechte vorgesehen sind, was, worauf im Weiteren noch einzugehen sein wird, u. a. staatliche Beschränkungen der internationalen Bewegungsfreiheit durchaus zulässt (s. unten).

6 Auf Ergebnisse dieser Forschung wird im Weiteren noch eingegangen.
} 
den Zutritt und den Aufenthalt auf ihrem Territorium zu entscheiden. Sozialwissenschaftlich ist diesbezüglich wiederholt überzeugend argumentiert worden, dass die diskriminierende Unterscheidung zwischen Staatsbürgern und Nicht-Staatsbürgern unter Bedingungen globaler Ungleichheiten eine der wirkungsmächtigsten Unterscheidungen ist, der ein gesellschaftsstrukturell verankertes Primat nationaler Interessen und eine primäre Verpflichtung nationalstaatlicher Politik auf die Interessen der Staatsbürger*innen entspricht (Kreckel 2006; Pogge 2011). Dass diese Form der Diskriminierung in völkerrechtlichen und nationalstaatlichen Gesetzgebungen von Diskriminierungsverboten ausgenommen wird, ist nicht zuletzt als Ausdruck des Interesses der relativ wohlhabenden Staaten zu erklären, unerwünschte Zuwanderung mit legalen Mitteln verhindern zu können. Bereits in der Allgemeinen Erklärung der Menschenrechte war dementsprechend, und bereits damals nachweisbar aus migrationspolitischen Gründen, ein Recht auf Bewegungsfreiheit nur innerhalb des Staates vorgesehen (AEDM, Art. 13), keine grenzüberschreitende Bewegungsfreiheit. Hierin wird exemplarisch deutlich, dass in politische und rechtliche Diskriminierungsverbote bzw. Fassungen der Rechte, die diskriminierungsfrei zu gewähren sind, immer auch nationalstaatlich gefasste Interessen eingehen, d.h. Interessen, die aus der politischen und rechtlichen Verfasstheit moderner Gesellschaften als $\mathrm{Na}-$ tionalstaaten innerhalb einer durch gravierende Ungleichheiten gekennzeichneten Weltgesellschaft resultieren (Stichweh 2000). Um unter diesen Bedingungen eine Situation aufrechterhalten zu können, die durch eine formale politische und rechtliche Gleichheit der Staatsbürger*innen sowie sozialstaatlich garantierte Mindeststandards innerhalb von Nationalstaaten einerseits, gravierende Ungleichheiten zwischen Nationalstaaten andererseits gekennzeichnet ist, ist die politische und rechtliche Inanspruchnahme der diskriminierenden Unterscheidung zwischen Staatsbürger*innen und Ausländer*innen nicht verzichtbar (Scherr 2015, 2020). Dies führt dazu, dass diese Unterscheidung im hegemonialen politischen Diskurs der Nationalstaaten nicht als Fall von Diskriminierung thematisiert wird, sondern als legitime Voraussetzung der gesellschaftlichen Ordnung gilt.

Erklärungsbedürftig ist auch, warum die in der Allgemeinen Erklärung der Menschenrechte genannte Kategorie der sozialen Herkunft im europäischen und deutschen Recht nicht als Diskriminierungskategorie anerkannt ist. Denn die sozialwissenschaftliche Forschung hat nicht nur immer wieder strukturelle Benachteiligungen nachgewiesen, die aus dem soziö̈konomischen Status, der sozialen Klassenlage und der Schichtungsposition resultieren. Vielmehr liegen auch Befunde dazu vor, dass dafür auch diskriminierende Gruppenkonstruktionen und Eigenschaftszuschreibungen an die Angehörigen der sozial benachteiligten Klassen und Schichten, etwa in der Form von Unterklassendiskursen, einflussreich waren und sind, die in der Diskriminierungsforschung als Ausprägungen von Klassismus bezeichnet werden (Kemper und Weinbach 2016). ${ }^{7}$ Bei einer Erklärung, weshalb klassistische Diskriminierung bislang gleichwohl nicht zu den politisch anerkannten und rechtlich institutionalisierten Diskriminierungskategorien gehört, ist erstens zu berücksichtigen, dass die Thematisierung von sozialen Ungleichheiten einerseits, Diskriminierung anderer-

\footnotetext{
7 Deren Auswirkungen auf Bildung und Berufschancen werden u. a. in der Form von Wohngebietseffekten sichtbar (El-Mafaalani et al. 2015).
} 
seits in politischen Diskursen, aber auch in den Sozialwissenschaften, weitgehend unabhängig voneinander erfolgt ist: Soziale Ungleichheiten wurden und werden nach wie vor primär als Effekte ökonomischer Strukturen in den Blick genommen, wobei die Frage, ob und ggf. wie diese historisch und systematisch mit Formen der Diskriminierung (Rassismus, Geschlecht) verschränkt sind, weitgehend ausgeklammert wurde. Zweitens ist festzustellen, dass für gegenwärtig einflussreiche politische und wissenschaftliche Diskurse eine identitätspolitische Ausrichtung kennzeichnend ist, die Forderungen nach der Anerkennung der Angehörigen diskriminierter Gruppen als gleichwertige und gleichberechtigte Gesellschaftsmitglieder ins Zentrum stellt und dabei auf rechtliche und diskursive Anerkennungsverhältnisse fokussiert ist; dagegen werden sozioökonomische Ungleichheiten und die Zusammenhänge von Klassenlagen mit diskriminierenden Unterscheidungen, die auch darin bestehen, dass direkte und indirekte Effekte sozioökonomischer Benachteiligung für die Plausibilisierung diskriminierender Zuschreibungen bedeutsam sind, vernachlässigt. Demgegenüber hat Nancy Fraser (2017) in m. E. überzeugender Weise dazu aufgefordert, die historischen und systematischen Wechselwirkungen zwischen sozioökonomischen Verteilungsverhältnissen und soziokulturellen Anerkennungsverhältnissen in den Blick zu nehmen sowie die politisch und wissenschaftlich etablierte Aufspaltung in sozialpolitische und identitätspolitische Perspektiven aufzubrechen.

Für eine darauf ausgerichtete Forschung lassen sich bereits in klassischen soziologischen Beiträgen zu Diskriminierungsforschung bedeutsame Anknüpfungspunkte finden. So hat etwa bereits Herbert Blumer $(1954,1961)$ bezogen auf Rassismus in überzeugender Weise argumentiert, dass diskriminierende Unterscheidungen immer auch Vorstellungen darüber umfassen, was die Positionen sozialer Gruppen in den gesellschaftlichen Hierarchien sein sollen, also Annahmen darüber, wem welche Rechte und welche Privilegien zustehen sollen. Norbert Elias (Elias 1987; Elias und Scotson 1993) hat auf Zusammenhänge zwischen diskriminierenden Gruppenkonstruktion und gesellschaftlichen „Positionskämpfen“ (Elias 1987, S. 27) hingewiesen. Seine Analyse akzentuiert, dass kollektive Eigenschaftszuschreibungen ein Element und eine Folge von Konflikten um Machtpositionen sind. Diese gewinnen ihre Plausibilität daraus, dass jeweiligen Außenseitern von den Etablierten soziale Positionen zugewiesen werden, deren Folgen der Anknüpfungspunkt vorurteilshafter Deutungen sind, welche die Auswirkungen sozialer Verhältnisse als vermeintliche Effekte der Eigenschaften der Angehörigen sozialer Gruppen darstellen. Soziologische Diskriminierungsforschung ist damit aufgefordert, Vorurteile nicht als letztliche Erklärungsebene für Diskriminierung zu beanspruchen und bezüglich der Verbreitung von Vorurteilen auf sozialpsychologische Konzepte zu rekurrieren, sondern zu analysieren, warum und wie Vorurteile und diskriminierende Praktiken als Bestandteile und Ergebnisse von Positionskämpfen in Strukturen sozialer Ungleichheit entstehen und wirkungsmächtig werden. ${ }^{8}$

\footnotetext{
8 Siehe dazu ausführlicher und zu weiteren diskriminierungssoziologischen Konzepten: Scherr (2017a).
} 


\subsection{Diskriminierung als Fremdbestimmung der Identität auf der Grundlage imaginärer Vergemeinschaftung}

In Zusammenhang mit diesen Hinweisen zur sozialen Genese von Vorurteilen ist hier noch auf eine weitere folgenreiche Einsicht der soziologischen Diskriminierungsforschung einzugehen: darauf, dass Diskriminierung als gesellschaftliches Phänomen nicht angemessen in Anlehnung an die sozialpsychologische Forschung zu Entstehung von Vorteilen in Konflikten zwischen Realgruppen verstanden werden kann (siehe dazu etwa Wagner 2020). Denn im Unterschied zu Realgruppenkonflikten um knappe Ressourcen und erstrebenswerte Güter, die sich zu Feindseligkeiten und vorurteilshaften Eigenschaftszuschreibungen zuspitzen können, existieren für gesellschaftlich bedeutsame Formen von Diskriminierung folgenreiche Zugehörigkeiten und Konflikte nicht unabhängig von diskursiven Gruppenkonstruktionen, die als Formen einer imaginären Vergemeinschaftung wirksam werden: In Anschluss u.a. an Webers Analyse von Ethnisierungsprozessen (Scherr 2000) und Andersons Konzept der Nation als imaginäre Gemeinschaft (Anderson 2006) ist davon auszugehen, dass Vorstellungen der Zugehörigkeit und Zusammengehörigkeit von Menschen, die sich nicht kennen und deren Lebensbedingungen sich in vielen Hinsichten unterscheiden, sich nicht naturwüchsig als Ergebnis gemeinsamer Praxis und geteilter Erfahrungen ergeben, sondern wirkungsmächtige Diskurse und Ideologien voraussetzen, die einer Selbst- und Fremdwahrnehmung auf der Grundlage dieser Imaginationen und damit einhergehenden Abgrenzungen und Annahmen über Gruppenkonflikte Plausibilität verleihen.

Brubaker (2007) hat diesbezüglich akzentuiert, dass die gesellschaftlich wirkungsmächtige Nivellierung der Unterschiede von Realgruppen und imaginären Gruppen auch in den Sozialwissenschaften einflussreich ist, wenn in sozialwissenschaftlichen Texten von ethnischen und religiösen Gruppen in einer Weise die Rede ist, die deren Existenz als gegebene soziale Gemeinschaften postuliert, für die (etwa auf der Grundlage statistischer Analysen, die Ethnizität als unabhängige Variable verwenden) gemeinsame Normen, Werte und Interessen angenommen werden und für die unterstellt wird, dass diejenigen, die nach jeweiligen Kriterien als Mitglieder oder Angehörige der jeweiligen imaginären Gruppe betrachtet werden, sich selbst auch als Angehörige dieser Gruppenkategorie definieren und eine darauf bezogene soziale Identität für sich reklamieren. Im vorliegenden Zusammenhang ist diese Kritik des Gruppismus in Alltagsdenken, medialen Wirklichkeitsbildern und den Sozialwissenschaften deshalb relevant, weil damit auch auf den direkten sowie über mediale und pädagogische Kommunikation vermittelten indirekten Beitrag der Sozialwissenschaften zur Verbreitung und Legitimation von Kategorien hingewiesen ist, die Grundlage von Diskriminierung sein können. Exemplarisch deutlich wird dies am Fall der Kategorie Migrationshintergrund: Diese war zunächst eine für Zwecke der statistischen Datenanalyse entwickelte Kategorie, die dann aber vermittelt über die Medien in die politische Kommunikation und in die Alltagskommunikation eingewandert ist und dort als Grundlage einer naturalisierenden Unterscheidung von Personen mit und ohne Migrationshintergrund wirksam werden kann, die dann diskriminierende Auswirkungen hat, wenn Personen mit Migrationshintergrund 
der Anspruch auf gleichberechtigte gesellschaftliche Zugehörigkeit bestritten wird (Foroutan 2019; Scherr 2014b).

Die Problematik der Diskriminierung beginnt jedoch, worauf zuerst Schütz (2011 [1957]) hingewiesen hat, nicht erst mit der diskriminierenden Verwendung von Gruppenkonstruktionen. Vielmehr unterläuft bereits die Zuschreibung einer vermeintlichen Gruppenzugehörigkeit den Anspruch von Personen, als selbstbestimmungsfähige Individuen wahrgenommen $\mathrm{zu}$ werden, und sie kann $\mathrm{zu}$ Beschädigungen der Identität führen: „Sie werden durch die bloße Auferlegung der Typisierung sich selbst entfremdet und wie austauschbare Repräsentanten von typisierten Merkmalen und Charakteristika behandelt" (ebd.: 215). Ersichtlich stimmt diese Überlegung mit Goffmans Untersuchung der Bedeutung von Stigmatisierung für die Entwicklung von Identitäten überein. In Anlehnung an Goffmans Analyse der „Techniken der Bewältigung beschädigter Identität“ (Goffman 1967, S. 1) ist soziologische Diskriminierungsforschung jedoch aufgefordert, von Stigmatisierung und Diskriminierung Betroffene nicht nur als passive Opfer von Identitätszuschreibungen in den Blick zu nehmen, sondern auch die individuellen und kollektiven Formen der aktiven Auseinandersetzung damit zu berücksichtigen. Auf der Grundlage der einschlägigen internationalen Forschung können diesbezüglich individuelle und kollektive sowie defensive (z.B. Vermeidung von Situation, in denen mit Diskriminierung gerechnet wird), pragmatische (z.B. stillschweigendes Aushalten diskriminierender Äußerungen) und offensive (z.B. Zurückweisung diskriminierender Äußerungen) unterschieden werden (Scherr und Breit 2019a). Dabei stellt es eine wichtige Aufgabe soziologischer Forschung dar, zu untersuchen, was die sozialen Bedingungen dafür sind, die jeweiligen Formen der individuellen oder kollektiven Auseinandersetzung zu ermöglichen, zu erleichtern oder zu erschweren. In einer international vergleichenden Studie haben Lamont et al. (2016) diesbezüglich insbesondere auf den Einfluss nationalgesellschaftlich einflussreicher kultureller Repertoires hingewiesen, die jeweilige Deutungen und Bewertungen von Diskriminierung und von darauf bezogenen Reaktionsweisen beeinflussen. Scherr und Breit (2019b) haben daran anschließend aufgezeigt, dass auch rechtliche Aufenthaltssicherheit in Verbindung mit Anerkennungserfahrungen in schulischen, beruflichen und informellen Kontexten im Fall von Geflüchteten eine wichtige soziale Grundlage dafür ist, dass Betroffene sich befähigt und berechtigt fühlen, sich selbstbewusst gegen Diskriminierung zur Wehr zu setzen. Bislang liegt der Schwerpunkt der empirischen soziologischen Diskriminierungsforschung jedoch auf der Untersuchung von diskriminierenden Strukturen und Praktiken in unterschiedlichen gesellschaftlichen Teilbereichen sowie Erhebungen, welche die gesellschaftliche Verbreitung diskriminierender Einstellungen in den Blick nehmen. Dagegen finden sich nur wenige Studien, die auf die Untersuchung der sozialen Bedingungen von Diskriminierungskritik oder die Verbreitung diskriminierungskritischer Einstellungen ausgerichtet sind. Allerdings ist in Bezug auf migrationsbezogene Diskriminierung inzwischen eine Perspektivenverschiebung festzustellen, die in Veröffentlichungen deutlich wird, die auf erfolgreiche Überwindung von gesellschaftlicher Benachteiligung, auf Integrationserfolge sowie auf Tendenzen in Richtung auf einen Bedeutungsverlust der Unterscheidung Einheimische/Migrant*innen hinweisen (El-Mafaalani 2018; Foroutan 2019; Genkova und Riecken 2020). 


\section{Folgerungen}

Der zentrale Impuls der Soziologie sozialer Probleme besteht in der Aufforderung, gesellschaftlich etablierte Problemdefinitionen nicht als fraglos-selbstverständliche Grundlage empirischer Forschung vorauszusetzen, sondern Prozesse der sozialen Problemkonstruktion, der Entwicklung und Durchsetzung spezifischer Problematisierungen und Entproblematisierungen zu analysieren. Im Vorstehenden wurde versucht aufzuzeigen, dass diese Perspektive auch für soziologische Forschung über Diskriminierung produktiv ist. Es konnte exemplarisch aufgezeigt werden, dass für das Selbstverständnis moderner Gesellschaften zwar der Anspruch einer generellen Überwindung diskriminierender Unterscheidungen konstitutiv ist, dass aber aus angebbaren Gründen nur bestimmte Formen von Diskriminierung als politisch und rechtlich anerkannte Fälle ungerechtfertigter Benachteiligung gelten. Weiter wurde akzentuiert, dass ein einflussreiches sozialpsychologisches Verständnis von Diskriminierung als Folge von Vorurteilen unzureichend ist. Eine eigenständige soziologische Diskriminierungsforschung, die nicht nur direkte und absichtsvolle Diskriminierung, sondern auch die Ausprägungen, Gründe und Ursachen von struktureller und organisationeller Diskriminierung berücksichtigt, ist bislang aber nur unzureichend entwickelt und hat auch nicht den Status einer institutionell anerkannten soziologischen Teildisziplin erreicht. Insbesondere besteht ein Bedarf an weiteren empirischen Studien, die differenziert in den Blick nehmen, welche Diskriminierungsmerkmale in den unterschiedlichen gesellschaftlichen Teilbereichen in welcher Weise bedeutsam sind und die dabei auch berücksichtigen, ob bzw. in welcher Weise die Eigenlogik der gesellschaftlichen Teilsysteme und ihrer Organisationen dazu geeignet ist, den Einfluss diskriminierende Unterscheidungen zu begrenzen.

Meine eigenen empirischen Forschungserfahrungen legen diesbezüglich erhebliche Skepsis gegenüber vorschnellen Generalisierungen nahe. Z.B. hat sich dort gezeigt, dass international operierende Industrieunternehmen erheblich stärker dazu motiviert und in der Lage sind, Verfahren einer nicht-diskriminierenden Personalauswahl zu implementieren, als an regionalen Märkten orientierte Kleinbetriebe, oder dass die Diskriminierung von kopftuchtragenden Muslima auch in sozialen Kontexten Akzeptanz findet, in denen beansprucht wird, Formen der geschlechtsbezogenen und rassistischen Diskriminierung abzulehnen. In unserer Forschung wurde z.B. auch deutlich, dass die Diskriminierung von Sinti und Roma in spezifischer Weise mit historischen und aktuellen Formen sozioökonomischer Benachteiligung und sozialräumlicher Ausgrenzung verschränkt sowie im Fall von deutschen Sinti anders als bei migrantischen Roma - zu einem erheblichen Misstrauen gegenüber den Institutionen der deutschen Mehrheitsgesellschaft geführt hat, wodurch spezifische Ausgangsbedingungen für Strategien zur Überwindung ihrer Diskriminierung gegeben sind. Damit ist exemplarisch das verdeutlicht, was mit den vorstehenden Überlegungen begründet werden sollte: Dass ein Bedarf an einer gesellschaftstheoretisch und problemsoziologisch informierten empirischen Forschung besteht, die sich auf die differenzierte Erforschung der Formen und Folgen von Diskriminierung sowie von individuellen und kollektiven Bewältigungs- und Gegenstrategien in unterschiedlichen sozialen Kontext einlässt. 
Funding Open Access funding enabled and organized by Projekt DEAL.

Open Access Dieser Artikel wird unter der Creative Commons Namensnennung 4.0 International Lizenz veröffentlicht, welche die Nutzung, Vervielfältigung, Bearbeitung, Verbreitung und Wiedergabe in jeglichem Medium und Format erlaubt, sofern Sie den/die ursprünglichen Autor(en) und die Quelle ordnungsgemäß nennen, einen Link zur Creative Commons Lizenz beifügen und angeben, ob Änderungen vorgenommen wurden.

Die in diesem Artikel enthaltenen Bilder und sonstiges Drittmaterial unterliegen ebenfalls der genannten Creative Commons Lizenz, sofern sich aus der Abbildungslegende nichts anderes ergibt. Sofern das betreffende Material nicht unter der genannten Creative Commons Lizenz steht und die betreffende Handlung nicht nach gesetzlichen Vorschriften erlaubt ist, ist für die oben aufgeführten Weiterverwendungen des Materials die Einwilligung des jeweiligen Rechteinhabers einzuholen.

Weitere Details zur Lizenz entnehmen Sie bitte der Lizenzinformation auf http://creativecommons.org/ licenses/by/4.0/deed.de.

\section{Literatur}

ADS/Antidiskriminierungsstelle des Bundes. 2017. Dritter Gemeinsamer Bericht der Antidiskriminierungsstelle des Bundes und der in ihrem Zuständigkeitsbereich betroffenen Beauftragten der Bundesregierung und des Deutschen Bundestages. Berlin: ADS.

Albrecht, Günter, und Axel Groenemeyer. (Hrsg.) 2012. Handbuch Soziale Probleme. Wiesbaden: Springer.

Anderson, Benedict. 2006. Imagined communities, 3. Aufl., London: Verso.

Beck, Ulrich. 1984. Jenseits von Klasse und Stand. In Reinhard Kreckel (Hrsg.): Soziale Ungleichheiten. Soziale Welt Sonderband 2. Göttingen: Otto Schwartz \& Co.

Bertelsmann Stiftung. 2018. Faktensammlung Diskriminierung. Kontext Einwanderungsgesellschaft. Gütersloh. https://www.bertelsmann-stiftung.de/fileadmin/files/Projekte/Migration_fair_gestalten/IB_ Faktensammlung_Diskriminierung_2018.pdf. Zugegriffen: 15. Mai 2020.

Bielefeldt, Heiner. 2010. Das Diskriminierungsverbot als Menschenrechtsprinzip. In Diskriminierung. Grundlagen und Forschungsergebnisse, Hrsg. Ulrike Hormel, Albert Scherr, 21-34. Wiesbaden: VS.

Blumer, Herbert. 1954. Reflections on theory of race relations. In Race relations in world perspective: Papers read at the conference on race relations in world perspective, Hrsg. Andrew W. Lind, 3-19. Honolulu, Westport: Greenwood Press.

Blumer, Herbert. 1961. Race prejudice as a sense of group position. In Race relations. Problems and theory, Hrsg. Jitsuichi Masuoka, Valien Preston, 217-227. New York: Libraries Press.

Brubaker, Rogers. 2007. Ethnizität ohne Gruppen. Hamburg: Hamburger Edition.

Davidson, Alastair. 2012. The immutable laws of mankind. The struggle for universal human rights. Dodrecht: Springer.

Eckel, Jan. 2009. Utopie der Moral, Kalkül der Macht. Archiv für Sozialgeschichte 49:437-484.

Eckel, Jan, und Samuel Moyn (Hrsg.). 2012. Moral für die Welt? Menschenrechtspolitik in den 1970er Jahren. Göttingen: Vandenhoeck \& Ruprecht.

El-Mafaalani, Aladin. 2018. Das Integrationsparadox. Köln: Kiepenheuer \& Witsch.

El-Mafaalani, Aladin, Sebastian Kurtenbach, und Klaus Peter Strohmeier. 2015. Auf die Adresse kommt es an ... Segregierte Stadtteile als Problem- und Möglichkeitsräume begreifen. Weinheim: Beltz Juventa.

Elias, Norbert. 1987. Engagement und Distanzierung. Frankfurt a.M.: Suhrkamp.

Elias, Norbert. 1991. Die Gesellschaft der Individuen. Frankfurt a.M.: Suhrkamp.

Elias, Norbert, und John L. Scotson. 1993. Etablierte und Außenseiter. Frankfurt a.M.: Suhrkamp.

Feagin, Joe R., und Douglas Lee Eckberg. 1980. Discrimination: motivation, action, effects, and context. Annual Review of Sociology 6(1):1-20.

Foroutan, Naike. 2019. Die postmigrantische Gesellschaft. Bielefeld: transcript.

Fraser, Nancy. 2017. Für eine neue Linke, oder: Das Ende des progressiven Neoliberalismus. Blätter für deutsche und internationale Politik 2:71-76.

Friese, Julia. 2019. Wer ,Fremdenfeindlichkeit‘ sagt, übernimmt die Sicht der Täter - Die meisten Gespräche über Rassismus scheitern. Drei Expertinnen erklären, wie es besser geht - und wann nicht miteinander reden auch eine Lösung ist. https://www.zeit.de/die-antwort/2019-03/rassismus-herkunftidentitaet-kommunikation. Zugegriffen: 15. Mai 2020. 
Genkova, Petia, und Andrea Riecken (Hrsg.). 2020. Handbuch Migration und Erfolg. Wiesbaden: Springer. Goffman, Erving. 1967. Stigma. Frankfurt a.M.: Suhrkamp.

Gomolla, Mechthild. 2017. Direkte und indirekte, institutionelle und strukturelle Diskriminierung. In Handbuch Diskriminierung, Hrsg. Albert Scherr, Aladin El-Mafaalani, und Gökçen Yüksel, 133-156. Wiesbaden: Springer VS.

Gomolla, Mechthild, und Frank-Olaf Radtke. 2012. Institutionelle Diskriminierung. Die Herstellung ethnischer Differenz in der Schule, 3. Aufl., Wiesbaden: VS.

Groenemeyer, Axel. 2012. Soziologie sozialer Probleme - Fragestellungen, Konzepte und theoretische Perspektive. In Handbuch soziale Probleme, Hrsg. Günter Albrecht, Axel Groenemeyer, 17-116. Wiesbaden: Springer VS.

Hálfdarnason, Gudmundur, und Vilhem Vilhelmsson. 2017. Historische Diskriminierungsforschung. In Handbuch Diskriminierung, Hrsg. Albert Scherr, Aladin El-Mafaalani, und Gökçen Yüksel, 25-38. Wiesbaden: Springer VS.

Heintz, Bettina. 2015. Die Weltgesellschaft und ihre Menschenrechte. In Menschenrechte in der Weltgesellschaft, Hrsg. Bettina Heintz, Britta Leisering, 21-64. Frankfurt a.M.: Campus.

Heitmeyer, Wilhelm. 1992. Die Gefahren des schwärmerischen Antirassismus. Das Argument 195:675-683.

Heitmeyer, Wilhelm. 2012. Gruppenbezogene Menschenfeindlichkeit. Bd. 10. Frankfurt a.M.: Suhrkamp.

Hirschauer, Stefan. 2014. Un/doing Differences. Die Kontingenz sozialer Zugehörigkeiten. Zeitschrift für Soziologie 43(3):170-191.

Hormel, Ulrike. 2007. Diskriminierung in der Einwanderungsgesellschaft. Wiesbaden: VS.

Hormel, Ulrike, und Albert Scherr. 2004. Bildung für die Einwanderungsgesellschaft. Wiesbaden: VS.

Hormel, Ulrike, und Albert Scherr (Hrsg.). 2010. Diskriminierung. Grundlagen und Forschungsergebnisse. Wiesbaden: VS.

Ignatieff, Michael. 2002. Die Politik der Menschenrechte. Hamburg: Europäische Verlagsanstalt.

Imdorf, Christian. 2017. Understanding discrimination in hiring apprentices: how training companies use ethnicity to avoid organisational trouble. Journal of Vocational Education \& Training 69(3):405-423.

Imdorf, Christian, und Albert Scherr. 2015. Chancengerechtigkeit und Diskriminierung beim Übertritt in die Berufsausbildung. In Equity - Diskriminierung und Chancengerechtigkeit im Bildungswesen, Hrsg. Andrea Haenni Hoti, 83-89. Bern: Informationsstelle IDES.

Japp, Klaus. 2015. Zur Funktion der Menschenrechte in der Weltgesellschaft - Niklas Luhmanns Grundrechte als Institution revisited. In Menschenrechte in der Weltgesellschaft, Hrsg. Bettina Heintz, Britta Leisering, 65-97. Frankfurt a.M.: Campus.

Kemper, Andreas, und Heike Weinbach. 2016. Klassismus. Eine Einführung. Münster: Unrast.

Koenig, Matthias. 2015. Neo-institutionalistische Weltgesellschaftstheorie und die Perspektiven einer historischen Soziologie der Menschenrechte. In Menschenrechte in der Weltgesellschaft, Hrsg. Bettina Heintz, Britta Leisering, 98-129. Frankfurt a.M.: Campus.

Kreckel, Reinhard. 2006. Soziologie der sozialen Ungleichheit im globalen Kontext. Halle: Der Hallesche Graureiher - Universität Halle.

Lamont, Michele, et al. (Hrsg.). 2016. Getting respect. Responding to stigma and discrimination in the United States, Brazil \& Israel. Princeton: Princeton University Press.

Legge, Sandra, und Jürgen Mansel. 2012. Ethnische Diskriminierung, Rassismus und gruppenbezogene Menschenfeindlichkeit. In Handbuch soziale Probleme, Hrsg. Günter Albrecht, Axel Groenemeyer, 494-548. Wiesbaden: VS.

Luhmann, Niklas. 1993a. Individuum, Individualität, Individualismus. In Gesellschaftsstruktur und Semantik, Bd. 3, 149-258. Frankfurt a.M.: Suhrkamp.

Luhmann, Niklas. 1993b. Gesellschaftsstruktur und Semantik. Bd. 1. Frankfurt a.M.: Suhrkamp.

Margalit, Avalit. 1998. Politik der Würde. München: Fest.

Meyer, John W., John Boli, und George M. Thomas. 2005. Ontologie und Rationalisierung im Zurechnungssystem der westlichen Kultur. In Weltkultur. Wie die westlichen Prinzipien die Welt durchdringen, Hrsg. John W. Meyer, 17-46. Frankfurt a.M.: Suhrkamp.

Möller, Kurt, und Florian Neuscheler (Hrsg.). 2018. Wer will die hier schon haben? Ablehnungshaltungen und Diskriminierung in Deutschland. Stuttgart: Kohlhammer.

Pärli, Kurt. 2017. Rechtswissenschaftliche Diskrimiminierungsforschung. In Handbuch Diskriminierung, Hrsg. Albert Scherr, Aladin El-Mafaalani, und Gökcen Yüksel, 101-115. Wiesbaden: Springer VS.

Parkin, Frank. 1984. Strategien sozialer Schließung und Klassenbildung. In Soziale Ungleichheiten Soziale Welt, Bd. Sonderband 2, Hrsg. Reinhard Kreckel, 121-136. Göttingen: Otto Schwartz.

Pogge, Thomas. 2011. Weltarmut und Menschenrechte. Berlin: de Gruyter.

Rommelspacher, Birgit. 1995. Dominanzkultur. Texte zu Fremdheit. Berlin: Orlando Frauenverlag. 
Scherr, Albert. 2000. Ethnisierung als Ressource und Praxis. Prokla - Zeitschrift für kritische Sozialwissenschaft 120:399-414.

Scherr, Albert. 2002. Soziale Arbeit und die nicht beliebige Konstruktion sozialer Probleme in der funktional differenzierten Gesellschaft. Soziale Probleme 1/2:73-94.

Scherr, Albert. 2014a. Diskriminierung und soziale Ungleichheiten. Erfordernisse und Perspektiven einer ungleichheitsanalytischen Fundierung von Diskriminierungsforschung und Antidiskriminierungsstrategien. Wiesbaden: Springer.

Scherr, Albert. 2014b. The construction of National identity in Germany: „Migration background“ as a political and scientific category. Toronto: Ryerson University. http://www.ryerson.ca/content/dam/rcis/ documents/RCIS_WP_Scherr_No_2013_2.pdf. Zugegriffen: 15. Mai 2020.

Scherr, Albert. 2015. Migration, Menschenrechte und die Grenzen der Demokratie. In Migration und Minderheiten in der Demokratie, Hrsg. Philipp Eigenmann, Thomas Geisen, und Tobias Studer, 45-62. Wiesbaden: Springer.

Scherr, Albert. 2017a. Soziologische Diskriminierungsforschung. In Handbuch Diskriminierung, Hrsg. Albert Scherr, Aladin El-Mafaalani, und Gökçen Yüksel, 39-58. Wiesbaden: Springer VS.

Scherr, Albert. 2017b. Rassismus, Post-Rassismus und Nationalismus. Erfordernisse einer differenzierten Kritik. Peripherie 37:222-249.

Scherr, Albert. 2019. Kriminalität und Kriminalisierung. Warum Klärungsversuche an Überabstraktion und impliziter Normativität scheitern. Soziale Probleme 30(1):3-7.

Scherr, Albert. 2020. Grenzziehungen: Soziologie der paradoxen Forderung nach offenen Grenzen. In Der Begriff des Flüchtlings. Rechtliche, moralische und politische Kontroversen, Hrsg. Daniel Kersting, Marcus Leuoth, 137-155. Stuttgart: Metzler.

Scherr, Albert, und Helen Breit. 2019a. Erfolgreiche Bewältigung von Diskriminierung. In Handbuch Migration und Erfolg, Hrsg. Petia Genkova, Andrea Riecken, 83-106. Wiesbaden: Springer.

Scherr, Albert, und Helen Breit. 2019b. Diskriminierung, Anerkennung und der Sinn für die eigene soziale Position. Weinheim: Beltz Juventa.

Scherr, Albert, Caroline Janz, und Stefan Müller. (2015): Diskriminierung in der beruflichen Bildung. Wiesbaden: Springer VS.

Scherr, Albert, Aladin El-Mafaalani, und Gökçen Yüksel (Hrsg.). 2017. Handbuch Diskriminierung. Wiesbaden: Springer VS.

Schütz, Alfred. 2011. Gleichheit und Sinnstruktur der sozialen Welt. In Relevanz und Handeln, Bd. 2, Hrsg. Alfred Schütz, 171-250. Konstanz: UVK. 1957/2011.

Steinert, Heinz. 1981. Widersprüche, Kapitalstrategien und Widerstand oder: Warum ich den Begriff soziale Probleme nicht mehr hören kann. Kriminalsoziologische Bibliografie 32/33:56-91.

Stichweh, Rudolf. 2000. Die Weltgesellschaft. Frankfurt a.M.: Suhrkamp.

Tajfel, Henry. 1982. Gruppenkonflikt und Vorurteil. Bern: Huber.

Tilly, Charles. 1998. Durable inequality. Berkeley: University of California Press.

Wagner, Ulrich. 2020. Diskriminierung zwischen Gruppen. In Handbuch Migration und Erfolg, Hrsg. Petia Genkova, Andrea Riecken, 71-82. Wiesbaden: Springer. 